\title{
Disruptive behaviors and early sexual intercourse: the GAZEL youth study
}

Cédric Galéra (a,b)*; Antoine Messiah (b); Maria Melchior (c); Jean-François Chastang (c); Gaelle Encrenaz (b); Emmanuel Lagarde (b); Gregory Michel (d); Manuel-Pierre Bouvard (a); Eric Fombonne (e)

(a) University Victor Segalen Bordeaux 2, Charles-Perrens hospital, Child Psychiatry Department, Bordeaux, France

(b) INSERM, U897, Centre de Recherche Epidémiologie et Biostatistique, Equipe prévention et prise en charge des traumatismes, Bordeaux, France

(c) INSERM, U687, Villejuif, F-94807, France ; Université Paris XI, IFR69, Villejuif, F94807, France

(d) University Victor Segalen Bordeaux 2, Department of Psychology, Bordeaux, France

(e) McGill University, Montreal Children's Hospital, Psychiatry Department, Montreal, Quebec, Canada

Correspondance Cédric Galéra, Pôle de pédopsychiatrie universitaire, Centre Hospitalier Charles-Perrens, 121 rue de la Béchade, 33076 Bordeaux, France

Telephone/Fax numbers: +33556561719/+3356561736

E-mail: cedric.galera@u-bordeaux2.fr 


\begin{abstract}
Sexual health-risk behaviors in disruptive children are poorly understood. In a longitudinal population-based sample, event-time analyses showed that subjects with high levels of conduct disorder symptoms, particularly in combination with simultaneously high levels of hyperactivity-inattention symptoms, exhibited the highest risk for earlier sexual activity compared to controls, suggesting the need for prevention.
\end{abstract}

\title{
Key words
}

Attention Deficit Hyperactivity Disorder

Conduct Disorder

Sex behavior 


\section{Introduction}

Disruptive behavior disorders of childhood, particularly attention-deficit hyperactivity disorder (ADHD) and conduct disorder (CD), are associated with long-term adverse outcomes including socio-occupational difficulties, psychiatric problems, and risk behaviors such as substance use, bullying, or driving risk-taking (Hill and Maughan, 2001; Spencer and Biederman, 2007). Both disorders are frequent with estimated prevalence of each disorder at approximately 5\%. ADHD and CD are highly comorbid and controversy remains about their respective independent contributions regarding negative outcomes. Whilst early sexual intercourse is a significant health-risk behavior problem that has been linked to unintended pregnancy, sexually transmitted diseases and HIV infection (Coker et al., 1994; O'Donnell et al., 2001), few data are available about patterns of sexual activity in youths with disruptive behaviors.

In the Pittsburgh ADHD longitudinal study (Flory et al., 2006) where sample cases were male referrals, ADHD predicted earlier initiation of sexual activity in univariate models. However, while the relationship of $\mathrm{CD}$ symptoms to risky sexual behaviors was examined among probands, the link between CD symptoms and age at first sexual intercourse was not specifically examined. In another predominantly male sample of clinical referrals (Barkley et al., 2006), participants with hyperactivity reported an earlier age at first sexual intercourse when compared to non-hyperactive counterparts. It is noteworthy that in multivariate analyses, CD symptoms but not hyperactivity symptoms were significantly associated with early sexual intercourse. Recently, in a clinical female sample, ADHD alone was not associated with sexual intercourse before age 16 (Monuteaux et al., 2007). Of note, in girls with ADHD, CD was predictive of early sexual intercourse. On the whole, these three recent surveys were based on relatively small and selected clinical samples. 
This study investigated the large community-based and gender-balanced GAZEL youth study sample to examine the links between childhood disruptive symptoms and age at first sexual intercourse in both genders, controlling for youth psychopathology, temperament and environmental variables. We hypothesized that CD symptoms, particularly when combined with hyperactivity-inattention symptoms, might be a strong risk factor for early sexual intercourse.

\section{Methods}

\subsection{Subjects}

The GAZEL Youth study was carried out in 1991 and 1999 in France. Participating youths were recruited via one of their parents who is participating voluntarily in a long-term epidemiological investigation of health amongst employees of France's National Electricity and Gas Company (the GAZEL cohort). The GAZEL Youth study sample was selected to be socio-demographically representative of French youths. The sample was stratified by SES and family size according to 1991 census data using the official social class codification system. Data were collected through mailed self-report questionnaires in 1991 (parental questionnaire) and at follow-up in 1999 (parental and youth questionnaires). At baseline, 2582 youths aged 4-18 years were included. At follow-up, 1268 parents (49.1\%) and 1148 youths (44.5\%) participated (complete data available for 1107 participants of whom 916 were older than 15). Follow-up participants and non-participants did not significantly differ regarding most baseline characteristics either for males (child/parental psychopathology, parental marital status) or for females (child/parental psychopathology, parental marital status, socioeconomic status (SES)). However, participants at follow-up were younger $(P<0.01)$ and more often females $(P<0.01)$ than non-participants. Parents of male participants had higher SES $(P=0.03)$. The methodological details are available elsewhere (Goldberg et al., 2007; Galéra et 
al., 2008; Galéra et al., 2009). The study was approved by the French National Committee for Data Protection. Table 1 provides the demographics of the sample. 215 males and 299 females had already had sexual intercourse.

\subsection{Measures}

The youth questionnaire allowed evaluation of lifetime sexual experimentation and age at first sexual intercourse ("Have you ever had sexual intercourse?" and "How old were you when you had your first sexual intercourse?"). In accordance with a recommendation of the ethical board that reviewed the protocol, sexual activity was only ascertained among youths older than 15. Childhood psychopathology was assessed at baseline through parental completion of the 118-item Child Behavior Checklist (CBCL) (Achenbach and Rescorla, 2001). We used the CBCL variables hyperactivity-inattention symptoms (HI-s) (11 items, $\alpha=0.74$ ), CD symptoms (16 items, $\alpha=0.68$ ), and anxious/depressed symptoms (16 items, $\alpha=0.80$ ). Whereas the CBCL has been validated in France and is widely used for practice and research purposes, CBCL scale norms are not available for French samples. Consequently, for each dimension, subjects were classified into two groups (high and low level of symptoms) based on the $90^{\text {th }}$ percentile of the scores distribution, which is the recommended cut-off to differentiate cases and non-cases in community samples. Using the baseline parental questionnaire, we constructed a variable assessing whether the participating youth had deviant peers (yes v. no). Child temperament was ascertained retrospectively between ages 7-10 years by the follow-up parental questionnaire through the Emotionality, Activity, Sociability, Shyness (EAS) questionnaire (Buss and Plomin, 1984). We derived the variables Emotionality, Activity, Shyness, and Sociability $(5$ items each; respective $\alpha$ : $0.75 / 0.71 / 0.76 / 0.66)$. When the log-linearity hypothesis was not respected, we dichotomized the variable at the $75^{\text {th }}$ percentile of the scores distribution. Parental variables were extracted 
from the adult GAZEL cohort study files: SES (blue collar $v$. intermediate/white collar), marital status (divorced/separated/widowed/single $v$. married/cohabiting), psychological problems (frequently depressed/treated for depression/sleep-related problems: yes $v$. no).

\subsection{Analyses}

We decided a priori to stratify the sample by gender since developmental trajectories might be different in each sex. For each gender we fitted proportional hazard regression models to determine predictors of time to first sexual intercourse (dependent variable: time between birth and age at first sexual intercourse). We created dummy variables to represent the status of distinct groups (referent: low levels of HI-s and CD-s; high levels of: HI-s alone, CD-s alone, HI-s and CD-s). As recommended by the Hosmer and Lemeshow multivariate modeling strategy, variables with $\mathrm{p}<0.25$ were entered into the initial models. Backwards selection (variables deleted when $\mathrm{p}>0.05$ ) with control for confounding factors was then conducted. Proportional hazards assumptions and multicollinearity were systematically checked. Owing to missing data in the outcome, we conducted sensitivity analyses to verify that our findings were robust under different hypotheses of distribution of these missing data. Since the youngest part of the sample was less likely to have experimented sexual intercourse, we conducted further analyses controlling for age (continuous) at follow-up. Data were analysed using the SAS software.

\section{Results}

Tables $2 \mathrm{a}$ and $2 \mathrm{~b}$ show correlations among variables of interest and covariates in males and females. With regard to the distinct groups of interest, the respective mean age at first sexual intercourse was 1/in males: low levels of HI-s and CD-s (N=338): 17.1 years (s.d.=1.8); high levels of HI-s alone $(\mathrm{N}=25)$ : 16.8 years (s.d.=2.5), high levels of CD-s alone 
$(\mathrm{N}=29)$ : 16 years $(\mathrm{s.d.}=2.2)$, high levels of HI-s and CD-s $(\mathrm{N}=28)$ : 15.7 years $($ s.d.=1.6) and 2/in females: low levels of HI-s and CD-s (N=404): 17.3 years (s.d.=1.9); high levels of HI-s alone $(\mathrm{N}=33)$ : 17.1 years (s.d.=1.7), high levels of $\mathrm{CD}$-s alone $(\mathrm{N}=28)$ : 17 years $($ s.d.=1.6), high levels of HI-s and CD-s $(\mathrm{N}=24)$ : 16.4 years (s.d.=1.4). Table 3 provides results of hazards analyses examining first sexual intercourse in both genders. SES, parental marital status and psychopathology, youth deviant peers and anxious/depressed symptoms were removed from backwards selections. HI-s alone was not associated with a significant risk of earlier first sexual intercourse either in males or females. CD-s alone was a significant predictor in males and a marginally significant predictor in females. In both genders, the group with a high level of HI-s and CD-s was the most potent predictor, with roughly twice the risk of earlier first sexual intercourse compared to participants with no disruptive symptoms. High activity was associated with earlier first sexual intercourse in males, whereas shyness exerted a protective effect in females. We observed no multicollinearity between study variables. The hazard risk estimates hardly changed in sensitivity analyses. Further analyses controlling for age showed the same pattern of results.

\section{Discussion}

This is one of the very few studies examining links between ADHD, CD and first sexual intercourse. Our findings suggest that youths with conduct disorder symptoms are likely to have sexual intercourse earlier than those with no psychopathology, particularly if they also experience symptoms of hyperactivity-inattention simultaneously. The strength of this investigation is its large population-based sample including males and females followed longitudinally. Although the main limitation is sample attrition, drop-out analyses revealed few differences between participants and non-participants. Nevertheless, not having data on parental externalizing psychopathology limited the study of the effects of attrition. Second, 
due to self-reporting, measurement bias might have arisen since participants may have misrepresented (by under or over reporting) their sexual activity and because parent-reported disruptive symptoms might not have been as accurate as youth self-reports. However, selfreport measures have been demonstrated to be reliable and are shown to involve less desirability bias than face-to-face questionnaires (Tourangeau and Yan, 2007). Third, the study's generalizability is limited by the selection of participants from socioeconomically advantaged backgrounds (all participants had a parent with stable employment and participating in an epidemiological cohort study). Fourth, participants were not formally diagnosed with ADHD or CD. However CBCL cut-offs provided rates close to what should be expected on the basis of the general population data. Finally, temperament was assessed retrospectively, introducing the possibility of memory bias. However, any error in measurement is likely to be minor as temperament tends to be stable over time.

Hyperactivity-inattention alone was not associated with earlier first sexual intercourse. Subjects with CD symptoms, both alone and in conjunction with simultaneously high levels of HI-s, exhibited the highest risk for earlier sexual activity. This pattern of results found in both males and females is consistent with studies examining the joint effect of ADHD and CD on subsequent outcomes. It possibly argues for the specificity of a combined clinical ADHDCD subtype (Lynam, 1998) displaying the highest levels of impulsivity and cognitive deficits, which may heighten the liability to early sexual intercourse and other risky behaviors. This association between youth disruptive problems and health-damaging behaviors may reflect not only a common genetic liability but also an influence of disruptive behaviors in increasing developmental exposure to high-risk environments (e.g. deviant peer, parental-child conflict or poor academic involvement), as suggested by Keyes et al (2007). Of note, among youths with high liability to health-damaging behaviors, substance use and early sexual intercourse may have bidirectional influences. Further research is needed to elucidate the processes 
underlying their interplay. Finally, our study extends prior findings documenting an association between temperament and health-risk behaviors by showing a positive link between activity and early sex in males and a protective effect of shyness in females (Wills et al., 2001).

In summary, this study is of interest for families, educators, health professionals and youths themselves by underscoring the importance of directing preventive psycho-educational interventions in youths with disruptive behaviors toward a large range of health-risk behaviors that tend to cluster together, among which sexual practices should be included.

\section{Acknowledgements}

The authors express their thanks to EDF-GDF, especially the Service des Etudes Médicales and the Service Général de Médecine de Contrôle, and to the "Caisse centrale d'action sociale du personnel des industries électrique et gazière". We also wish to acknowledge the Postprofessional Risks - Cohorts team at INSERM U687 - CNAMTS, which manages the GAZEL cohort. Additionally, we are grateful to Pâquerette Goldberg from INSERM Unit 687. The GAZEL Cohort Study was funded by EDF-GDF and INSERM, and received grants from the Association de la Recherche sur le Cancer and the Fondation de France. The GAZEL Youth study was funded by grants from France's Ministry of Health (Direction Générale de la Santé) and from the Interdepartmental Mission for the Fight against Drugs and Drug addiction (MILDT) to Eric Fombonne. 


\section{References}

Achenbach, T.M., Rescorla, L.A., 2001. Manual for the ASEBA School-Age Forms and Profiles. Burlington, VT: University of Vermont, Research Center for Children, Youth, and Families.

Barkley, R.A., Fischer, M., Smallish, L., Fletcher, K., 2006. Young adult outcome of hyperactive children: adaptive functioning in major life activities. Journal of the American Academy of Child and Adolescent Psychiatry 45, 192-202.

Buss, A., Plomin, R., 1984. Temperament: early developing personality traits. London: Lawrence Erlbaum Associates.

Coker, A.L., Richter, D.L., Valois, R.F., McKeown, R.E., Garrison, C.Z., Vincent, M.L., 1994. Correlates and consequences of early initiation of sexual intercourse. The Journal of School Health 64, 372-377.

Flory, K., Molina, B.S., Pelham, W.E., Gnagy, E., Smith, B., 2006. Childhood ADHD predicts risky sexual behavior in young adulthood. Journal of Clinical Child and Adolescent Psychology 35, 571-577.

Galéra, C., Bouvard, M.P., Messiah, A., Fombonne, E., 2008. Hyperactivity-inattention symptoms in childhood and substance use in adolescence: the youth gazel cohort. Drug and Alcohol Dependence 94, 30-37.

Galéra, C., Melchior, M., Chastang, J.F., Bouvard, M.P., Fombonne, E. 2009. Childhood and adolescent hyperactivity-inattention symptoms and academic achievement 8 years later: the GAZEL Youth study. Psychological Medicine 39:1895-1906.

Goldberg, M., Leclerc, A., Bonenfant, S., Chastang, J.F., Schmaus, A., Kaniewski, N., Zins, M., 2007. Cohort profile: the GAZEL Cohort Study. International Journal of Epidemiology $36,32-39$. 
Hill, J., Maughan, B., 2001. Conduct disorders in childhood and adolescence. Cambridge, UK: Cambridge university press.

Keyes, M.A., Iacono, W.G., McGue, M. 2007. Early onset problem behavior, young adult psychopathology, and contextual risk. Twin Research and Human Genetics 10:45-53.

Lynam, D.R., 1998. Early identification of the fledgling psychopath: locating the psychopathic child in the current nomenclature. Journal of Abnormal Psychology 107, 566575.

Monuteaux, M.C., Faraone, S.V., Michelle Gross, L., 2007. Biederman J. Predictors, clinical characteristics, and outcome of conduct disorder in girls with attention-deficit/hyperactivity disorder: a longitudinal study. Psychological Medicine 37, 1731-1741.

O'Donnell, L., O'Donnell, C.R., Stueve, A., 2001. Early Sexual Initiation and Subsequent Sex-Related Risks Among Urban Minority Youth: The Reach for Health Study. Family Planning Perspective 33, 268-275.

Spencer, J., Biederman, J., Mick, E., 2007. Attention-deficit/hyperactivity disorder: diagnosis, lifespan, comorbidities, and neurobiology. Journal of Pediatric Psychology 32, 631-642. Tourangeau, R., Yan, T., 2007. Sensitive questions in surveys. Psychological Bulletin 33, $859-883$.

Wills, T.A., Cleary, S., Filer, M., Shinar, O., Mariani, J., Spera, K., 2001. Temperament related to early-onset substance use: test of a developmental model. Prevention Science 2, 145-163. 
Table 1. Sample characteristics

\section{Males $(\mathrm{N}=421) \quad$ Females $(\mathrm{N}=495)$}

Parental characteristics at baseline

Socioeconomic status

-Blue collar

14.5

17.4

-Medium

61.3

57.6

-White

24.2

25.0

Marital status

-Married/cohabiting

93.7

93.6

-Divorced/separated/widowed/single

6.3

6.4

Psychopathology

-Yes

11.4

14.0

$-\mathrm{No}$

88.6

86.0

Youth characteristics at follow-up

Age, years

$19.9(15.0-25.7) \quad 20.2(15.0-25.9)$

Residency

-Rural

17.0

15.8

-2000-30000 inhabitants

41.5

42.5

-Over 30000 inhabitants

41.5

41.7

Occupation

-Student

80.3

81.0

-Worker

13.3

12.8

-Without

4.0

3.6

-Other

2.4

2.6

Values given are percentage or mean (range) 
Table 2a. Correlations among variables of interest and covariates in males

\begin{tabular}{lllllllll}
\hline & 1 & 2 & 3 & 4 & 5 & 6 & 7 & 8 \\
\hline 1. HI-s & 1 & & & & & & & \\
2. CD-s & $0.53^{*}$ & 1 & & & & & & \\
3. AD-s & $0.54^{*}$ & $0.36^{*}$ & 1 & & & & & \\
4. Emotionality & $0.33^{*}$ & $0.29^{*}$ & $0.34^{*}$ & 1 & & & & \\
5. Activity & 0.14 & 0.09 & -0.06 & 0.02 & 1 & & & \\
6. Shyness & 0.11 & 0.11 & $0.30^{*}$ & $0.29 *$ & $-0.42^{*}$ & 1 & & \\
7. Sociability & 0.02 & 0.03 & $-0.16^{*}$ & 0.07 & $0.45^{*}$ & $-0.49 *$ & 1 & \\
8. Age at $1^{\text {st }}$ sex & $-0.20^{*}$ & $-0.22^{*}$ & -0.13 & -0.03 & $-0.23^{*}$ & 0.07 & -0.06 & 1 \\
\hline
\end{tabular}

Table $2 \mathrm{~b}$. Correlations among variables of interest and covariates in females

\begin{tabular}{lllllllll}
\hline & 1 & 2 & 3 & 4 & 5 & 6 & 7 & 8 \\
\hline 1. HI-s & 1 & & & & & & & \\
2. CD-s & $0.50^{*}$ & 1 & & & & & & \\
3. AD-s & $0.41^{*}$ & $0.20^{*}$ & 1 & & & & & \\
4. Emotionality & $0.27^{*}$ & $0.23^{*}$ & $0.26^{*}$ & 1 & & & & \\
5. Activity & 0.04 & 0.03 & $-0.20^{*}$ & $-0.14^{*}$ & 1 & & & \\
6. Shyness & 0.07 & 0.09 & $0.27^{*}$ & $0.26^{*}$ & $-0.52^{*}$ & 1 & & \\
7. Sociability & 0.08 & 0.01 & $-0.16^{*}$ & 0.04 & $0.53^{*}$ & $-0.58^{*}$ & 1 & \\
8. Age at $1^{\text {st }}$ sex & -0.02 & $-0.14^{*}$ & 0.09 & -0.04 & -0.07 & 0.08 & -0.01 & 1 \\
\hline
\end{tabular}

Child Behavior Checklist (HI-s: hyperactivity-inattention symptoms, CD-s: conduct disorder symptoms, AD-s: anxious-depressed symptoms)

Age at $1^{\text {st }}$ sex: age at first sexual intercourse $*: p<0.05$ 
Table 3. Childhood hyperactivity-inattention and conduct disorder symptoms, temperament and age at first sexual intercourse by gender

\begin{tabular}{lccc}
\hline & $\begin{array}{c}\text { Fully adjusted Hazard Ratios [95\%CI] } \\
\text { Males }\end{array}$ & Females \\
\cline { 2 - 3 } $\begin{array}{lcc}\text { HI-s and CD-s } \\
\text { Low HI-s and Low CD-s }\end{array}$ & 1.00 & 1.00 \\
High HI-s only & $1.07[0.59-1.93]$ & & $1.31[0.82-2.15]$ \\
High CD-s only & $1.71[1.04-2.83]$ & & $1.57[0.95-2.58]$ \\
High HI-s and High CD-s & $2.33[1.36-3.99]$ & & $1.81[1.11-2.95]$ \\
Other covariates & & \\
Temperament: activity & $1.73[1.27-2.35]$ & \\
Temperament: shyness & ---- & $0.73[0.55-0.96]$ \\
\hline
\end{tabular}

HI-s: hyperactivity-inattention symptoms, CD-s: conduct disorder symptoms Fully adjusted model in males $(\mathrm{N}=386)$ : wald $\chi^{2}=23.64(P<0.0001)$

Fully adjusted model in females $(\mathrm{N}=458)$ : wald $\chi^{2}=14.25(P=0.0065)$ 